PROCEEDINGS OF THE

AMERICAN MATHEMATICAL SOCIETY

Volume 132, Number 3, Pages 911-918

S 0002-9939(03)07121-1

Article electronically published on September 18, 2003

\title{
REMARK ABOUT THE SPECTRUM OF THE $p$-FORM LAPLACIAN UNDER A COLLAPSE WITH CURVATURE BOUNDED BELOW
}

\author{
JOHN LOTT \\ (Communicated by Jozef Dodziuk)
}

\begin{abstract}
We give a lower bound on the number of small positive eigenvalues of the $p$-form Laplacian in a certain type of collapse with curvature bounded below.
\end{abstract}

\section{INTRODUCTION}

A general problem in spectral geometry is to estimate the eigenvalues of the $p$-form Laplacian on a closed Riemannian manifold $M$ in terms of the geometry of $M$. From Hodge theory, the number of zero eigenvalues is $\mathrm{b}_{p}(M)$, the $p$-th Betti number of $M$. Hence the issue is to understand the positive eigenvalues. The papers [2], 7] and 8] study the case when one assumes an upper bound on the diameter of the manifold and double-sided bounds on the sectional curvatures. An important phenomenon is the possible appearance of positive eigenvalues of the $p$ form Laplacian that approach zero as a manifold collapses with bounded curvature.

The analysis of 7$]$ and 8 uses the results of Cheeger, Fukaya and Gromov on the geometric structure of manifolds that collapse with double-sided curvature bounds. If one only assumes a lower sectional curvature bound, then there are some structure results about collapsing in [4] and [12, but the theory is less developed than in the bounded curvature case.

In this paper we look at the small positive eigenvalues of the $p$-form Laplacian in an example of collapse with curvature bounded below. Namely, suppose that a compact Lie group $G$ acts isometrically on $M$ on the left. Give $G$ a left-invariant Riemannian metric. For $\epsilon>0$, let $\epsilon G$ denote $G$ with its Riemannian metric multiplied by $\epsilon^{2}$. Let $M_{\epsilon}$ denote $M=G \backslash(\epsilon G \times M)$ equipped with the quotient Riemannian metric $g_{\epsilon}$, where $G$ acts diagonally on $\epsilon G \times M$ on the left. If $G$ is connected, then $\lim _{\epsilon \rightarrow 0} M_{\epsilon}=G \backslash M$ in the Gromov-Hausdorff topology, and as $\epsilon$ goes to zero, the sectional curvatures of $M_{\epsilon}$ stay uniformly bounded below [12].

For notation, if $M$ is a smooth connected closed manifold with Riemannian metric $g$, let $\left\{\lambda_{p, j}(M, g)\right\}_{j=1}^{\infty}$ denote the eigenvalues (counted with multiplicity) of the Laplacian on $\overline{\operatorname{Im}(d)} \subset \Omega_{L^{2}}^{p}(M)$. The projection $M \rightarrow G \backslash M$ induces a map $\mathrm{H}^{p}(G \backslash M ; \mathbb{R}) \rightarrow \mathrm{H}^{p}(M ; \mathbb{R})$.

Received by the editors September 19, 2002 and, in revised form, November 3, 2002.

2000 Mathematics Subject Classification. Primary 58G25; Secondary 53C23.

Research supported by NSF grant DMS-0072154. 
Theorem 1.1. If $j=\operatorname{dim}\left(\operatorname{Ker}\left(\mathrm{H}^{p}(G \backslash M ; \mathbb{R}) \rightarrow \mathrm{H}^{p}(M ; \mathbb{R})\right)\right)$, then

$$
\lim _{\epsilon \rightarrow 0} \lambda_{p, j}\left(M_{\epsilon}, g_{\epsilon}\right)=0 \text {. }
$$

In Section 2 we prove Theorem 1.1. The main points of the proof are the use of a certain variational expression for $\lambda_{p, j}(M, g)$, due to Cheeger and Dodziuk [3], and the avoidance of dealing with the detailed orbit structure of the group action. We then look at the example of an $S^{1}$-action on $S^{2 n}$, which is the suspension of the Hopf action of $S^{1}$ on $S^{2 n-1}$, and show that our results slightly improve those of Takahashi [10]. In Section 3 we make some further remarks.

I thank Junya Takahashi for sending me a copy of his paper.

\section{Proof of Theorem 1.1}

Let $\mathfrak{g}$ be the Lie algebra of $G$. It acquires an inner product from the left-invariant Riemannian metric on $G$. Given $\mathfrak{x} \in \mathfrak{g}$, let $\mathfrak{X}$ be the corresponding vector field on $M$. Let $i_{\mathfrak{X}}$ denote interior multiplication by $\mathfrak{X}$.

Let $\Omega^{*}(M)$ denote the smooth differential forms on $M$. Let $\Omega_{L^{2}}^{*}(M)$ be the $L^{2}$-completion of $\Omega^{*}(M)$. Put

$$
\Omega_{\text {max }}^{*}(M)=\left\{\omega \in \Omega_{L^{2}}^{*}(M): d \omega \in \Omega_{L^{2}}^{*+1}(M)\right\},
$$

where $d \omega$ is originally defined distributionally.

Put

$$
\Omega_{G}^{*}(M)=\left\{\omega \in \Omega^{*}(M): g \cdot \omega=\omega \text { for all } g \in G\right\}
$$

and

$$
\Omega_{\text {basic }}^{*}(M)=\left\{\omega \in \Omega_{G}^{*}(M): i_{\mathfrak{X}} \omega=0 \text { for all } \mathfrak{x} \in \mathfrak{g}\right\} .
$$

Let $\Omega_{G, L^{2}}^{*}(M)$ and $\Omega_{b a s i c, L^{2}}^{*}(M)$ be the $L^{2}$-completions of $\Omega_{G}^{*}(M)$ and $\Omega_{b a s i c}^{*}(M)$, respectively. Put

$$
\Omega_{\text {basic }, \max }^{*}(M)=\left\{\omega \in \Omega_{\text {basic }, L^{2}}^{*}(M): d \omega \in \Omega_{\text {basic }, L^{2}}^{*+1}(M)\right\},
$$

where $d \omega$ is originally defined distributionally. Then $\Omega_{\text {basic,max }}^{*}(M)$ is a complex.

From [6] and 11, the cohomology of the complex $\Omega_{\text {basic }}^{*}(M)$ is isomorphic to $\mathrm{H}^{*}(G \backslash M ; \mathbb{R})$.

Lemma 2.1. The cohomology of the complex $\Omega_{\text {basic,max }}^{*}(M)$ is also isomorphic to $\mathrm{H}^{*}(G \backslash M ; \mathbb{R})$.

Proof. The proof is essentially the same as that of 11]. For $U$ an open subset of $G \backslash M$, let $\widehat{U}$ be its preimage in $M$. For $p \geq 0$, put $S^{p}(U)=\Omega_{b a s i c, \text { max }}^{p}(\widehat{U})$. If $V$ is an open subset of $U$, then there is an obvious restriction homomorphism $S^{p}(U) \rightarrow S^{p}(V)$. We obtain a fine sheaf $\mathbf{S}^{p}$ over $G \backslash M$. If $\mathbf{R}$ denotes the constant sheaf on $G \backslash M$ with fiber $\mathbb{R}$, then we have a complex of sheaves

$$
\mathbf{R} \longrightarrow \mathbf{S}^{0} \longrightarrow \mathbf{S}^{1} \longrightarrow \mathbf{S}^{2} \longrightarrow \ldots
$$

From sheaf cohomology theory, it suffices to prove that (2.5) is a resolution of $\mathbf{R}$. As in [11], one can use the slice theorem to reduce this to proving the middle exactness of the complex

$$
\Omega_{\text {basic,max }}^{p-1}(N) \longrightarrow \Omega_{\text {basic }, \max }^{p}(N) \longrightarrow \Omega_{\text {basic,max }}^{p+1}(N) .
$$

Here $N$ is a Euclidean space and "basic" refers to a Lie group $H$ that acts linearly and isometrically on $N$. As in [11], one can use a homotopy operator $A$ to prove 
the exactness. The only point to note is that the homotopy operator $A$ used in [1] sends $\Omega_{b a s i c, \max }^{*}$ to itself.

The quotient map $p: \epsilon G \times M \rightarrow M_{\epsilon}$ defines a principal $G$-bundle. Pullback gives an isomorphism $p^{*}: \Omega^{*}\left(M_{\epsilon}\right) \cong \Omega_{\text {basic }}^{*}(\epsilon G \times M)$. The parallelism of $G$ gives an isomorphism

$$
\Omega^{*}(\epsilon G \times M) \cong C^{\infty}(G) \otimes \Lambda^{*}\left(\mathfrak{g}^{*}\right) \otimes \Omega^{*}(M) .
$$

Taking $G$-invariants gives isomorphisms

$$
\Omega_{G}^{*}(\epsilon G \times M) \rightarrow\left(C^{\infty}(G) \otimes \Lambda^{*}\left(\mathfrak{g}^{*}\right) \otimes \Omega^{*}(M)\right)^{G} \stackrel{\beta}{\rightarrow} \Lambda^{*}\left(\mathfrak{g}^{*}\right) \otimes \Omega^{*}(M),
$$

where $\beta$ comes from the map that sends $\sum_{k} f_{k} \otimes \eta_{k} \otimes \omega_{k} \in C^{\infty}(G) \otimes \Lambda^{*}\left(\mathfrak{g}^{*}\right) \otimes \Omega^{*}(M)$ to $\sum_{i} f_{k}(e) \eta_{k} \otimes \omega_{k} \in \Lambda^{*}\left(\mathfrak{g}^{*}\right) \otimes \Omega^{*}(M)$.

Let $\left\{\mathfrak{x}_{j}\right\}_{j=1}^{\operatorname{dim}(G)}$ be a basis of $\mathfrak{g}$. For $\mathfrak{x} \in \mathfrak{g}$, let $e\left(\mathfrak{x}^{*}\right)$ denote exterior multiplication by $\mathfrak{x}^{*}$ on $\Lambda^{*}\left(\mathfrak{g}^{*}\right)$.

Lemma 2.2. There is an isomorphism of complexes $\mathcal{I}: \Omega^{*}(M) \rightarrow \Omega_{\text {basic }}^{*}(\epsilon G \times$ $M) \subset \Lambda^{*}\left(\mathfrak{g}^{*}\right) \otimes \Omega^{*}(M)$ given by

$$
\begin{aligned}
\mathcal{I}(\sigma) & =\left(\prod_{j=1}^{\operatorname{dim}(G)}\left(1-e\left(\mathfrak{x}_{j}^{*}\right) \otimes i_{\mathfrak{X}_{j}}\right)\right)(1 \otimes \sigma) \\
& =\sum_{k=0}^{\operatorname{dim}(G)}(-1)^{k} \sum_{1 \leq j_{1}<\ldots<j_{k} \leq \operatorname{dim}(G)}\left(\mathfrak{x}_{j_{k}}^{*} \wedge \ldots \wedge \mathfrak{x}_{j_{1}}^{*}\right) \otimes i_{\mathfrak{X}_{j_{1}}} \ldots i_{\mathfrak{X}_{j_{k}}} \sigma .
\end{aligned}
$$

Proof. If $\sum_{k} f_{k} \otimes \eta_{k} \otimes \omega_{k} \in\left(C^{\infty}(G) \otimes \Lambda^{*}\left(\mathfrak{g}^{*}\right) \otimes \Omega^{*}(M)\right)^{G}$ is $G$-basic, then for $\mathfrak{x} \in \mathfrak{g}$, we also have

$$
\sum_{k}\left(f_{k} \otimes i_{\mathfrak{r}} \eta_{k} \otimes \omega_{k}+(-1)^{\left|\eta_{k}\right|} f_{k} \otimes \eta_{k} \otimes i_{\mathfrak{X}} \omega_{k}\right)=0 .
$$

Then

$$
\sum_{k}\left(f_{k}(e) i_{\mathfrak{x}} \eta_{k} \otimes \omega_{k}+(-1)^{\left|\eta_{k}\right|} f_{k}(e) \eta_{k} \otimes i_{\mathfrak{X}} \omega_{k}\right)=0,
$$

i.e., if $\sum_{k} \eta_{k} \otimes \omega_{k}$ lies in the image of $\beta$ restricted to $\Omega_{\text {basic }}^{*}(\epsilon G \times M)$, then

$$
\sum_{k}\left(i_{\mathfrak{x}} \eta_{k} \otimes \omega_{k}+(-1)^{\left|\eta_{k}\right|} \eta_{k} \otimes i_{\mathfrak{x}} \omega_{k}\right)=0 .
$$

It follows that $\sum_{k} \eta_{k} \otimes \omega_{k}$ can be written as $\mathcal{I}(\sigma)$ for some $\sigma \in \Omega^{*}(M)$. Thus $\mathcal{I}$ is surjective. It is clearly injective.

It remains to show that $\mathcal{I}$ is a morphism of complexes. Let $d^{i n v}$ denote the (finitedimensional) differential on $\Lambda^{*}\left(\mathfrak{g}^{*}\right)$. If an element of $\Omega_{G}^{*}(\epsilon G \times M)$ is represented as $\sum_{k} f_{k} \otimes \eta_{k} \otimes \omega_{k} \in C^{\infty}(G) \otimes \Lambda^{*}\left(\mathfrak{g}^{*}\right) \otimes \Omega^{*}(M)$, then the $G$-invariance implies that for $\mathfrak{x} \in \mathfrak{g}$,

$$
\sum_{k}\left(\mathfrak{x} f_{k} \otimes \eta_{k} \otimes \omega_{k}+f_{k} \otimes \eta_{k} \otimes \mathcal{L}_{\mathfrak{X}} \omega_{k}\right)=0
$$


The differential of $\sum_{k} f_{k} \otimes \eta_{k} \otimes \omega_{k}$ is represented by

$$
\begin{aligned}
& \sum_{k}\left(\sum_{j=1}^{\operatorname{dim}(G)} \mathfrak{x}_{j} f_{k} \otimes e\left(\mathfrak{x}_{j}^{*}\right) \eta_{k} \otimes \omega_{k}+f_{k} \otimes d^{i n v} \eta_{k} \otimes \omega_{k}\right. \\
& \left.\quad+(-1)^{\left|\eta_{k}\right|} f_{k} \otimes \eta_{k} \otimes d \omega_{k}\right)
\end{aligned}
$$

From (2.13), this equals

$$
\begin{aligned}
\sum_{k}( & -\sum_{j=1}^{\operatorname{dim}(G)} f_{k} \otimes e\left(\mathfrak{x}_{j}^{*}\right) \eta_{k} \otimes \mathcal{L}_{\mathfrak{X}_{j}} \omega_{k}+f_{k} \otimes d^{i n v} \eta_{k} \otimes \omega_{k} \\
& \left.+(-1)^{\left|\eta_{k}\right|} f_{k} \otimes \eta_{k} \otimes d \omega_{k}\right) .
\end{aligned}
$$

Using $\beta$, it follows that the induced differential on $\Lambda^{*}\left(\mathfrak{g}^{*}\right) \otimes \Omega^{*}(M)$ sends $\sum_{k} \eta_{k} \otimes \omega_{k}$ to

$$
\sum_{k}\left(-\sum_{j=1}^{\operatorname{dim}(G)} e\left(\mathfrak{x}_{j}^{*}\right) \eta_{k} \otimes \mathcal{L}_{\mathfrak{X}_{j}} \omega_{k}+d^{i n v} \eta_{k} \otimes \omega_{k}+(-1)^{\left|\eta_{k}\right|} \eta_{k} \otimes d \omega_{k}\right)
$$

One can check that when this acts on $\mathcal{I}(\sigma)$, the result is $\mathcal{I}(d \sigma)$. Thus $\mathcal{I}$ is an isomorphism of complexes.

In fact, under our identifications, $\mathcal{I}$ is the same as $p^{*}$.

Let $M^{r e g}$ be the union of the principal orbits for the $G$-action on $M$. It is a dense open subset of $M$ with full measure. If $m \in M^{r e g}$, let $H \subset G$ be its isotropy subgroup, with Lie algebra $\mathfrak{h}$. Define $\alpha: \mathfrak{g} \rightarrow T_{m} M$ by $\alpha(\mathfrak{x})=\mathfrak{X}_{m}$. It passes to an injection $\bar{\alpha}: \mathfrak{g} / \mathfrak{h} \rightarrow T_{m} M$. For $\epsilon \geq 0$, put $\rho_{\epsilon}(m)=\operatorname{det}^{1 / 2}\left(\epsilon^{2} \mathrm{Id} .\left.\right|_{\mathfrak{g} / \mathfrak{h}}+\bar{\alpha}^{*} \bar{\alpha}\right)$. If $m \notin M^{r e g}$, put $\rho_{\epsilon}(m)=0$. Note that for $\epsilon>0, \rho_{\epsilon}^{-1}(m)<\rho_{0}^{-1}(m)$.

Lemma 2.3. $\rho_{0}^{-1} \in L^{1}(M, d \mathrm{vol})$.

Proof. If $m \in M^{r e g}$, then up to an overall constant, $\rho_{0}(m)$ is the volume of the orbit $G \cdot m$. Then $\int_{M^{\text {reg }}} \rho_{0}^{-1}(m) d \operatorname{vol}(m)$ is proportionate to the volume of $G \backslash M^{\text {reg }} \subset$ $G \backslash M$, which is seen to be finite.

Let $\left\{\mathfrak{x}_{j}\right\}_{j=1}^{\operatorname{dim}(G)}$ be an orthonormal basis of $\mathfrak{g}$.

Lemma 2.4. For $\epsilon>0$, there is a positive constant $C(\epsilon)$ such that $\Omega^{*}\left(M_{\epsilon}\right)$ is isometrically isomorphic to $\Omega^{*}(M)$ with the new norm

$$
\begin{aligned}
\|\omega\|_{\epsilon}^{2}= & C(\epsilon) \int_{M} \rho_{\epsilon}^{-1}(m)\left(|\omega(m)|_{M}^{2}\right. \\
& \left.+\sum_{k=1}^{\operatorname{dim}(G)} \epsilon^{-2 k} \sum_{1 \leq j_{1}<\ldots<j_{k} \leq \operatorname{dim}(G)}\left|i_{\mathfrak{X}_{j_{1}}} \ldots i_{\mathfrak{X}_{j_{k}}} \omega(m)\right|_{M}^{2}\right) d \operatorname{vol}(m) .
\end{aligned}
$$

Proof. We can compute the norm squared of $\omega \in \Omega^{*}\left(M_{\epsilon}\right)$ by taking the local norm squared of $p^{*} \omega$ on $\epsilon G \times M^{r e g}$, dividing by the function that assigns to 
$(g, m) \in \epsilon G \times M^{r e g}$ the volume of the orbit $G \cdot(g, m)$, and integrating over $\epsilon G \times M^{r e g}$. If $m \in M^{r e g}$, then the relative volume of $G \cdot(g, m)$ is

$$
\operatorname{det}^{1 / 2}\left(\epsilon^{2} \text { Id. }\left.\right|_{\mathfrak{g}}+\alpha^{*} \alpha\right)=\epsilon^{\operatorname{dim}(H)} \rho_{\epsilon}(m) .
$$

The map $\beta$ of (2.8) is an isometry, up to a constant. Since $\left\{\epsilon^{-1} \mathfrak{x}_{j}\right\}_{j=1}^{\operatorname{dim}(G)}$ is an orthonormal basis for $T_{e}(\epsilon G)$, the lemma follows from Lemma 2.2 .

Proof of Theorem 1.1. Put $\lambda_{p, j}(\epsilon)=\lambda_{p, j}\left(M_{\epsilon}, g_{\epsilon}\right)$. From [3],

$$
\lambda_{p, j}(\epsilon)=\inf _{V} \sup _{\eta \in V-0} \sup _{\theta \in d^{-1}(\eta)} \frac{\|\eta\|_{\epsilon}^{2}}{\|\theta\|_{\epsilon}^{2}}
$$

where $V$ ranges over the $j$-dimensional subspaces of $\operatorname{Im}\left(d: \Omega^{p-1}(M) \rightarrow \Omega^{p}(M)\right)$, and $\theta \in d^{-1}(\eta) \subset \Omega^{p-1}(M)$.

Take $j=\operatorname{dim}\left(\operatorname{Ker}\left(\mathrm{H}^{p}(G \backslash M ; \mathbb{R}) \rightarrow \mathrm{H}^{p}(M ; \mathbb{R})\right)\right)$. From Lemma2.1 the inclusion of complexes $\Omega_{\text {basic }}^{*}(M) \rightarrow \Omega_{\text {basic,max }}^{*}(M)$ induces an isomorphism on cohomology. Then there is a $j$-dimensional subspace $V$ of

$$
\operatorname{Ker}\left(d: \Omega_{\text {basic }}^{p}(M) \rightarrow \Omega_{\text {basic }}^{p+1}(M)\right) \cap \operatorname{Im}\left(d: \Omega^{p-1}(M) \rightarrow \Omega^{p}(M)\right)
$$

such that if $\eta \in V-0$, then $\eta \notin \operatorname{Im}\left(d: \Omega_{\text {basic,max }}^{p-1}(M) \rightarrow \Omega_{\text {basic, } L^{2}}^{p}(M)\right)$. We claim that

$$
\lim _{\epsilon \rightarrow 0} \sup _{\eta \in V-0} \sup _{\theta \in d^{-1}(\eta)} \frac{\|\eta\|_{\epsilon}^{2}}{\|\theta\|_{\epsilon}^{2}}=0 .
$$

This will suffice to prove the theorem.

Suppose that (2.21) is not true. Then there are a constant $c>0$, a sequence $\left\{\epsilon_{r}\right\}_{r=1}^{\infty}$ in $\mathbb{R}^{+}$approaching zero, a sequence $\left\{\eta_{r}\right\}_{r=1}^{\infty}$ in $V-0$ and a sequence $\left\{\theta_{r}\right\}_{r=1}^{\infty}$ in $\Omega^{p-1}(M)$ such that for all $r, d \theta_{r}=\eta_{r}$ and

$$
\frac{\left\|\eta_{r}\right\|_{\epsilon_{r}}^{2}}{\left\|\theta_{r}\right\|_{\epsilon_{r}}^{2}} \geq c
$$

Doing a Fourier decomposition of $\theta_{r}$ with respect to $G$, the ratio in (2.22) does not decrease if we replace $\theta_{r}$ by its $G$-invariant component. Thus we may assume that $\theta_{r}$ is $G$-invariant.

Without loss of generality, we can replace the norm $\|\cdot\|_{\epsilon}$ of (2.17) by the same norm divided by $C(\epsilon)$, which we again denote by $\|\cdot\|_{\epsilon}$. Since $\eta_{r}$ is smooth on $M$, it follows from Lemma 2.3 that the function $\rho_{0}^{-1}(m)\left|\eta_{r}(m)\right|_{M}^{2}$ is integrable on $M$. Without loss of generality, we may assume that

$$
\int_{M} \rho_{0}^{-1}(m)\left|\eta_{r}(m)\right|_{M}^{2} d \operatorname{vol}(m)=1 .
$$

Since $\left\{\eta_{r}\right\}_{r=1}^{\infty}$ lies in the sphere of a finite-dimensional space, there will be a subsequence, which we relabel as $\left\{\eta_{r}\right\}_{r=1}^{\infty}$, that converges smoothly to some $\eta_{\infty} \in V-0$.

From (2.22),

$$
\begin{aligned}
\left\|\theta_{r}\right\|_{\epsilon_{r}}^{2} & \leq c^{-1}\left\|\eta_{r}\right\|_{\epsilon_{r}}^{2}=c^{-1} \int_{M} \rho_{\epsilon_{r}}^{-1}(m)\left|\eta_{r}(m)\right|_{M}^{2} d \operatorname{vol}(m) \\
& \leq c^{-1} \int_{M} \rho_{0}^{-1}(m)\left|\eta_{r}(m)\right|_{M}^{2} d \operatorname{vol}(m)=c^{-1} .
\end{aligned}
$$


For large $r$,

$$
\begin{aligned}
\int_{M}\left|\theta_{r}(m)\right|_{M}^{2} d \operatorname{vol}(m) & \leq\left(\inf _{M} \rho_{\epsilon_{r}}^{-1}\right)^{-1} \int_{M} \rho_{\epsilon_{r}}^{-1}(m)\left|\theta_{r}(m)\right|_{M}^{2} d \operatorname{vol}(m) \\
& \leq\left(\inf _{M} \rho_{1}^{-1}\right)^{-1} c^{-1} .
\end{aligned}
$$

We now work with respect to the metric $g$ on $M$. By weak-compactness of the unit ball in $L^{2}$, there is a subsequence of $\left\{\theta_{r}\right\}_{r=1}^{\infty}$, which we relabel as $\left\{\theta_{r}\right\}_{r=1}^{\infty}$, that converges weakly in $L^{2}$ to some $\theta_{\infty} \in \Omega_{G, L^{2}}^{p-1}(M)$. Then for $\sigma \in \Omega^{p}(M)$,

$$
\begin{aligned}
\left\langle\sigma, \eta_{\infty}\right\rangle_{M}-\left\langle d^{*} \sigma, \theta_{\infty}\right\rangle_{M} & =\lim _{r \rightarrow \infty}\left(\left\langle\sigma, \eta_{r}\right\rangle_{M}-\left\langle d^{*} \sigma, \theta_{r}\right\rangle_{M}\right) \\
& =\lim _{r \rightarrow \infty}\left\langle\sigma, \eta_{r}-d \theta_{r}\right\rangle_{M}=0 .
\end{aligned}
$$

Thus $\theta_{\infty} \in \Omega_{\max }^{p-1}(M)$ and $d \theta_{\infty}=\eta_{\infty}$.

From (2.24), we also obtain that for each $1 \leq j \leq \operatorname{dim}(G)$,

$$
\begin{aligned}
\int_{M}\left|i_{\mathfrak{X}_{j}} \theta_{r}(m)\right|_{M}^{2} d \operatorname{vol}(m) & \leq\left(\inf _{M} \rho_{\epsilon_{r}}^{-1}\right)^{-1} \int_{M} \rho_{\epsilon_{r}}^{-1}(m)\left|i_{\mathfrak{X}_{j}} \eta_{r}(m)\right|_{M}^{2} d \operatorname{vol}(m) \\
& \leq\left(\inf _{M} \rho_{1}^{-1}\right)^{-1} c^{-1} \epsilon_{r}^{2}
\end{aligned}
$$

Then for all $\sigma \in \Omega^{p-2}(M)$,

$$
\left\langle\sigma, i_{\mathfrak{X}_{j}} \theta_{\infty}\right\rangle_{M}=\left\langle\left(i_{\mathfrak{X}_{j}}\right)^{*} \sigma, \theta_{\infty}\right\rangle_{M}=\lim _{r \rightarrow \infty}\left\langle\left(i_{\mathfrak{X}_{j}}\right)^{*} \sigma, \theta_{r}\right\rangle_{M}=\lim _{r \rightarrow \infty}\left\langle\sigma, i_{\mathfrak{X}_{j}} \theta_{r}\right\rangle_{M}=0 .
$$

Thus $i_{\mathfrak{X}_{j}} \theta_{\infty}=0$ and $\theta_{\infty} \in \Omega_{\text {basic,max }}^{p-1}(M)$. Hence

$$
\eta_{\infty} \in \operatorname{Im}\left(d: \Omega_{\text {basic }, \max }^{\text {-1 }}(M) \rightarrow \Omega_{\text {basic }, L^{2}}^{p}(M)\right),
$$

which is a contradiction.

Example. Let $G=U(1)$ act on $M=S^{2 n}$ by the suspension of the Hopf action of $U(1)$ on $S^{2 n-1}$. Then $G \backslash M=U(1) \backslash S^{2 n}$ is the suspension of $\mathbb{C} P^{n-1}$. One finds that $\operatorname{Ker}\left(\mathrm{H}^{p}(G \backslash M ; \mathbb{R}) \rightarrow \mathrm{H}^{p}(M ; \mathbb{R})\right)$ is nonzero if and only if $p \in\{3,5, \ldots, 2 n-1\}$. From Theorem [1.1, as $\epsilon \rightarrow 0$, there are small eigenvalues of the $p$-form Laplacian on $\overline{\operatorname{Im}(d)} \subset \Omega_{L^{2}}^{p}\left(M_{\epsilon}\right)$ for $p \in\{3,5, \ldots, 2 n-1\}$. From the Hodge decomposition, there will also be small eigenvalues of the $p$-form Laplacian on $\overline{\operatorname{Im}\left(d^{*}\right)} \subset \Omega_{L^{2}}^{p}\left(M_{\epsilon}\right)$ for $p \in\{2,4, \ldots, 2 n-2\}$. Then using Hodge duality, one concludes that there are small eigenvalues on

1. $\overline{\operatorname{Im}\left(d^{*}\right)} \subset \Omega_{L^{2}}^{1}\left(M_{\epsilon}\right)$,

2. $\overline{\operatorname{Im}(d)} \subset \Omega_{L^{2}}^{p}\left(M_{\epsilon}\right)$ and $\overline{\operatorname{Im}\left(d^{*}\right)} \subset \Omega_{L^{2}}^{p}\left(M_{\epsilon}\right)$ for $p \in\{2,3,4, \ldots, 2 n-3,2 n-2\}$, and

3. $\overline{\operatorname{Im}(d)} \subset \Omega_{L^{2}}^{2 n-1}\left(M_{\epsilon}\right)$.

This slightly sharpens [10, Theorem 1.2]. Note that from eigenvalue estimates for the scalar Laplacian [1], there are no small eigenvalues on $\overline{\operatorname{Im}\left(d^{*}\right)} \subset \Omega_{L^{2}}^{0}\left(M_{\epsilon}\right)$, $\overline{\operatorname{Im}(d)} \subset \Omega_{L^{2}}^{1}\left(M_{\epsilon}\right), \overline{\operatorname{Im}\left(d^{*}\right)} \subset \Omega_{L^{2}}^{2 n-1}\left(M_{\epsilon}\right)$ or $\overline{\operatorname{Im}(d)} \subset \Omega_{L^{2}}^{2 n}\left(M_{\epsilon}\right)$.

\section{REMARKS}

1. In the case of a locally-free torus action, there is some intersection between Theorem[1.1] and the results of [2], [7] and [8. In [8] one deals with the cohomology of a certain $\mathbb{Z}$-graded sheaf $\mathrm{H}^{*}\left(A_{[0]}^{\prime}\right)$ on the limit space $X$. In the case of a collapsing coming from a locally-free torus action, Theorem 1.1 is a statement about the case 
$*=0$, when the sheaf $\mathrm{H}^{0}\left(A_{[0]}^{\prime}\right)$ is the constant $\mathbb{R}$-sheaf on $X$. Of course, the result of Theorem 1.1 will generally not give all of the small positive eigenvalues that arise in a collapse. As seen in the Example, one can obtain more small eigenvalues just from Hodge duality.

2. Theorem 1.1 indicates that the relevant cohomology of the limit space is the ordinary cohomology, as opposed for example to the $L^{2}$-cohomology. This is consistent with the results of $[8]$ in the bounded curvature case.

3. If $G$ has positive dimension and acts effectively on $M$, then Theorem 1.1 describes small positive eigenvalues in a collapsing situation. In some noncollapsing situations, one can show that small eigenvalues do not exist. Here is one such criterion.

Proposition 1. Let $\mathcal{M}$ be a collection of closed n-dimensional Riemannian manifolds, with $n>0$. Give $\mathcal{M}$ the Lipschitz metric, coming from bi-Lipschitz homeomorphisms. Suppose that $\mathcal{M}$ can be covered by a finite number of metric balls. For $p \in \mathbb{Z} \cap[0, n]$ and $j \geq 0$, there are positive numbers $a_{p, j}$ and $A_{p, j}$ so that if $(M, g) \in \mathcal{M}$, then $a_{p, j} \leq \lambda_{p, j}(M, g) \leq A_{p, j}$, and $\lim _{j \rightarrow \infty} a_{p, j}=\infty$.

Proof. Suppose first that for some $p$ and $j$, there is no upper bound on $\lambda_{p, j}(M, g)$ as $(M, g)$ ranges over $\mathcal{M}$. Then there is a sequence $\left\{\left(M_{i}, g_{i}\right)\right\}_{i=1}^{\infty}$ in $\mathcal{M}$ with the property that $\lim _{i \rightarrow \infty} \lambda_{p, j}\left(M_{i}, g_{i}\right)=\infty$. A subsequence of $\left\{\left(M_{i}, g_{i}\right)\right\}_{i=1}^{\infty}$, which we relabel as $\left\{\left(M_{i}, g_{i}\right)\right\}_{i=1}^{\infty}$, will have finite distance from some $\left(M_{\infty}, g_{\infty}\right) \in \mathcal{M}$. Then there are a number $\epsilon \geq 0$ and a sequence of bi-Lipschitz homeomorphisms $h_{i}: M_{\infty} \rightarrow M_{i}$ so that for all $i$,

$$
e^{-\epsilon} g_{\infty} \leq h_{i}^{*} g_{i} \leq e^{\epsilon} g_{\infty} .
$$

Here $h_{i}^{*} g_{i}$ is a Lipschitz metric on $M_{\infty}$. From Hodge theory,

$$
\lambda_{p, j}\left(M_{i}, g_{i}\right)=\inf _{V} \sup _{\eta \in V-0} \sup _{\theta \in d^{-1}(\eta)} \frac{\|\eta\|_{M_{i}}^{2}}{\|\theta\|_{M_{i}}^{2}}
$$

where $V$ ranges over the $j$-dimensional subspaces of $\operatorname{Im}\left(d: \Omega_{\max }^{p-1}\left(M_{i}\right) \rightarrow \Omega_{L^{2}}^{p}\left(M_{i}\right)\right)$, and $\theta \in d^{-1}(\eta) \subset \Omega_{m a x}^{p-1}\left(M_{i}\right)$. By naturality,

$$
\lambda_{p, j}\left(M_{i}, g_{i}\right)=\inf _{V} \sup _{\eta \in V-0} \sup _{\theta \in d^{-1}(\eta)} \frac{\|\eta\|_{h_{i}^{*} g_{i}}^{2}}{\|\theta\|_{h_{i}^{*} g_{i}}^{2}}
$$

where $V$ ranges over the $j$-dimensional subspaces of $\operatorname{Im}\left(d: \Omega_{\max }^{p-1}\left(M_{\infty}\right) \rightarrow\right.$ $\left.\Omega_{L^{2}}^{p}\left(M_{\infty}\right)\right)$, and $\theta \in d^{-1}(\eta) \subset \Omega_{\max }^{p-1}\left(M_{\infty}\right)$.

As in [3, it follows from (3.1) and (3.3) that there is a positive integer $J$ which only depends on $n$ so that

$$
e^{-J \epsilon} \lambda_{p, j}\left(M_{\infty}, g_{\infty}\right) \leq \lambda_{p, j}\left(M_{i}, g_{i}\right) \leq e^{J \epsilon} \lambda_{p, j}\left(M_{\infty}, g_{\infty}\right) .
$$

This contradicts the assumption that $\lim _{i \rightarrow \infty} \lambda_{p, j}\left(M_{i}, g_{i}\right)=\infty$.

Now suppose that it is not true that there is a uniform lower bound $a_{p, j}$ on $\left\{\lambda_{p, j}(M, g)\right\}_{(M, g) \in \mathcal{M}}$ with the property that $\lim _{j \rightarrow \infty} a_{p, j}=\infty$. Then there are a number $C>0$, a sequence $\left\{\left(M_{i}, g_{i}\right)\right\}_{i=1}^{\infty}$ in $\mathcal{M}$ and a sequence of integers $\left\{j_{i}\right\}_{i=1}^{\infty}$ such that $\lim _{i \rightarrow \infty} j_{i}=\infty$ and for each $i, \lambda_{p, j_{i}}\left(M_{i}, g_{i}\right) \leq C$. Take a subsequence $\left\{\left(M_{i}, g_{i}\right)\right\}_{i=1}^{\infty}$ and an $\left(M_{\infty}, g_{\infty}\right)$ as before. Then for each $j$,

$$
\lambda_{p, j}\left(M_{\infty}, g_{\infty}\right) \leq \sup _{i \rightarrow \infty} \lambda_{p, j_{i}}\left(M_{\infty}, g_{\infty}\right) \leq \sup _{i \rightarrow \infty} e^{J \epsilon} \lambda_{p, j_{i}}\left(M_{i}, g_{i}\right) \leq e^{J \epsilon} C .
$$


This contradicts the fact that the spectrum of the $p$-form Laplacian on $\left(M_{\infty}, g_{\infty}\right)$ is discrete.

Proposition 1 shows that in a certain sense, one has uniform eigenvalue bounds in the noncollapsing case. It seems possible that for a given $n \in \mathbb{Z}^{+}, K \in \mathbb{R}$ and $v, D>$ 0 , the collection $\mathcal{M}$ of connected $n$-dimensional Riemannian manifolds $(M, g)$ with sectional curvatures greater than $K$, volume greater than $v$ and diameter less than $D$ satisfies the hypotheses of Proposition 1 It is known that there is a finite number of homeomorphism types in $\mathcal{M}[5]$. On the other hand, the analogous space of metrics defined with Ricci curvature, instead of sectional curvature, will generally not satisfy the hypotheses of Proposition 10 .

\section{REFERENCES}

[1] P. Bérard, From vanishing theorems to estimating theorems: the Bochner technique revisited, Bull. Amer. Math. Soc. 19 (1988), 371-406. MR 89i:58152

[2] B. Colbois and G. Courtois, A note on the first nonzero eigenvalue of the Laplacian acting on p-forms, Manuscripta Math. 68 (1990), 143-160. MR 91g:58290

[3] J. Dodziuk, Eigenvalues of the Laplacian on forms, Proc. Amer. Math. Soc. 85 (1982), 437443. MR 84k:58223

[4] K. Fukaya and T. Yamaguchi, The fundamental groups of almost nonnegatively curved manifolds, Ann. of Math. 136 (1992), 253-333. MR 93h:53041

[5] K. Grove, P. Petersen and J. Wu, Geometric finiteness theorems via controlled topology, Invent. Math. 99 (1990), 205-213. MR 90k:53075

[6] J. Koszul, Sur certains groupes de transformations de Lie, Géométrie differentielle, Colloques Internationaux du CNRS, Strasbourg (1953), pp. 137-141. MR 15:600g

[7] J. Lott, Collapsing and the differential form Laplacian: the case of a smooth limit space, Duke Math. J. 114 (2002), 267-306. MR 2003e:58047

[8] J. Lott, Collapsing and the differential form Laplacian: the case of a singular limit space, preprint, http://www.math.lsa.umich.edu/ lott.

[9] G. Perelman, Construction of manifolds of positive Ricci curvature with big volume and large Betti numbers, Comparison Geometry, Math. Sci. Res. Inst. Publ. 30, Cambridge Univ. Press, Cambridge (1997), pp. 157-163. MR 98h:53062

[10] J. Takahashi, Small eigenvalues on p-forms for collapsings of the even-dimensional spheres, Manuscripta Math. 109 (2002), 63-71. MR 2003j:58049

[11] A. Verona, A de Rham type theorem for orbit spaces, Proc. Amer. Math. Soc. 104 (1988), 300-302. MR 89j:57033

[12] T. Yamaguchi, Collapsing and pinching under a lower curvature bound, Ann. of Math. 133 (1991), 317-357. MR 92b:53067

Department of Mathematics, University of Michigan, Ann Arbor, Michigan 481091109

E-mail address: lott@umich.edu 